\title{
Rotationally resolved photoelectron angular distributions in resonance enhanced multiphoton ionization of NO
}

\author{
H. Rudolph and V. McKoy \\ Arthur Amos Noyes Laboratory of Chemical Physics, California Institute of Technology, Pasadena, \\ California 91125
}

(Received 18 April 1989; accepted 5 May 1989)

\begin{abstract}
We report calculated ionic rotational branching ratios and associated photoelectron angular distributions for $\left(1+1^{\prime}\right)$ resonance enhanced multiphoton ionization (REMPI) via the $R_{21}$ (20.5), $P_{21}+Q_{11}(25.5)$, and $P_{11}(22.5)$ branches of the $A^{2} \Sigma^{+}(3 s \sigma)$ state of NO. The branching ratios are dominated by even angular momentum transfer peaks, in agreement with the $\Delta N+l=$ odd $\left(\Delta N \equiv N_{+}-N_{i}\right.$ ) selection rule. Whereas the calculated photoelectron angular distributions are very branch dependent due to alignment, the ionic branching ratios are found to be less so. The present calculated results agree well with the experimental results of Allendorf et al.
\end{abstract}

\section{INTRODUCTION}

Resonance enhanced multiphoton ionization (REMPI), coupled with high-resolution photoelectron spectroscopy, has in recent years proven to be an important state-specific probe of the photoionization dynamics of molecular excited states. While vibrationally resolved spectra have been measured for many systems with conventional photoelectron spectroscopy, ${ }^{1,2}$ rotational resolution has been achieved in just a few cases. ${ }^{3-5}$ When combined with photoelectron angular detection such rotationally resolved spectra provide a very detailed dynamical picture of the photoionization process. For example, the photoelectron angular measurements parallel and perpendicular to the polarization vector by Reilly et $a l .{ }^{5}$ in rotationally resolved REMPI via the $D^{2} \Sigma^{+}(3 p \sigma)$ state of NO, made it possible to explicitly identify the surprisingly large $p$-wave character of the photoelectron wave function.

The preceding paper ${ }^{6}$ reports the results of measurements of photoelectron angular distributions and ionic rotational branching ratios for $\left(1+1^{\prime}\right)$ REMPI via the $A^{2} \Sigma^{+}(3 s \sigma)$ state of $\mathrm{NO}^{7}$ In this paper we present calculated rotational branching ratios along with photoelectron angular distributions associated with these same REMPI processes. This work extends our earlier studies ${ }^{8-15}$ where we addressed underlying dynamical features associated with measurements of rotationally resolved REMPI spectra of the ${ }^{2} \Sigma^{+}$Rydberg states of NO., ${ }^{4,5,15,16}$ These features included: (i) ion rotational distributions which manifest the significant non-atomic-like character of molecular photoelectron orbitals, e.g., a strong $\Delta N=0$ peak in the rotational branching ratios seen in photoionization of the essentially atomic $3 p$ orbital of the $D^{2} \Sigma^{+}$state of NO, implying a $3 p \rightarrow k p$ transition in accordance with the $\Delta N+l=$ odd selection rule, ${ }^{11,14,15}$ (ii) dependence of ion rotational branching ratios on intermediate state alignment, ${ }^{12}$ and (iii) strong dependence of rotational branching ratios on $J$ at low $J$ values of the resonant state. ${ }^{13}$

Our ionic rotational branching ratios and photoelectron angular distributions are calculated at a photoelectron energy of $\approx 180 \mathrm{meV}$ for $\left(1+1^{\prime}\right)$ REMPI via the $R_{21}(20.5)$,
$P_{21}+Q_{11}$ (25.5), and $P_{11}(22.5)$ branches of the $A^{2} \Sigma^{+}$state of NO. The photoelectron angular distributions are branch dependent with branching ratios symmetric around $\Delta N=0$ for the high $J$ branches accessed in the present study $(J \cong 20.5$ ). This is in contrast to the behavior at low $J$ where the branching ratios show a strong asymmetry and dependence on $J .^{13,16}$ The calculated branching ratios and photoelectron angular distributions are found to agree well with the experimental results of Allendorf et al., ${ }^{6,7}$ although some disagreement is seen for the $\Delta N=-2$ signal of the $R_{21}(20.5)$ branch.

\section{THEORY}

The theoretical method used in the present studies has been described previously. ${ }^{9,17}$ Here we only discuss a few pertinent details. The $\left(1+1^{\prime}\right)$ REMPI process of interest here is viewed as a one-photon excitation from an initially unaligned (all $M_{J}$ levels equally populated) ground state $\left(X^{2} \Pi\right)$ to an aligned resonant intermediate state $\left(A^{2} \Sigma^{+}\right)$ with subsequent one-photon ionization out of this state,

$$
\begin{aligned}
\mathrm{NO} & X^{2} \Pi\left(v_{0}=0, J_{0}\right) \stackrel{h v}{\rightarrow} \mathrm{NO}^{*} A^{2} \Sigma^{+}\left(v_{i}=0, J_{i}, N_{i}\right) \\
& \stackrel{h v}{\rightarrow} \mathrm{NO}^{+} X^{1} \Sigma^{+}\left(v_{+}=0, J_{+}=N_{+}\right)+e^{-(k) .}
\end{aligned}
$$

For linearly polarized light and in the absence of collisions and other $M_{J}$-mixing interactions, each $M_{J}$ level can be treated as an independent ionization channel. For the present weak-field studies the relative population of the $M_{J}$ levels $\left(\rho_{i i}\right)$ of the intermediate state is adequately described by

$$
\rho_{i i} \propto\left(\begin{array}{lll}
J_{0} & 1 & J_{i} \\
M_{i} & 0 & M_{i}
\end{array}\right)^{2} A,
$$

where $A$ is the line strength given in Ref. 18. The probability $P(\theta, \phi)$ of photoelectron ejection in the direction $(\theta, \phi)$ with respect to the laser polarization vector $(\epsilon)$ is then given by an incoherent sum over both the photoionization probability $\Gamma_{i}$ of the individual $M_{J}$ levels and rotational branches (for mixed branches), 


$$
\begin{aligned}
P(\theta, \phi) & =\sum_{\text {branches }} \sum_{i} \rho_{i i} \Gamma_{i}\left(J_{i}, N_{i}, N_{+}\right) \\
& =\sum_{L=0}^{L_{\max } / 2} \beta_{2 L} P_{2 L}(\cos \theta) .
\end{aligned}
$$

In the summation on the righ-hand side of Eq. (3) the photoelectron angular distributions are expressed as a weighted sum over Legendre polynomials of even order. $L_{\max }$ is determined by the dynamics of the particular REMPI process and is 4 for the present $\left(1+1^{\prime}\right)$ low intensity high-J experiments. In the evaluation of Eq. (3) it is assumed that the polarization vectors of the two photons in the $\left(1+1^{\prime}\right)$ REMPI scheme are parallel. It is simple to extend Eq. (2) to allow for an arbitrary angle between the vectors. In the following we assume the lasers to be polarized parallel to each other unless otherwise specified. Changing the angle between the polarization vectors of the excitation and ionization lasers simply probes the dependence of the cross sections (and angular distributions) on the alignment of the intermediate state.

We assume the $X^{2} \Pi$ state to be intermediate between Hund's case (a) and Hund's case (b), and the resonant ${ }^{2} \Sigma^{+}$ and final ${ }^{1} \Sigma^{+}$states to be adequately described by Hund's case (b) coupling. In the present frozen-core and orbital approximation, ${ }^{9}$ the photoionization probability for a particular $M_{J}$ level $\Gamma_{i}$ is proportional to $\langle f|\mu| i\rangle^{2}$, the square of the one-electron matrix element between the final continuum orbital $|f\rangle$ and the resonant Rydberg orbital $|i\rangle$. For the REMPI process of Eq. (1) $\langle f|\mu| i\rangle$ can then be written as $^{13}$

$$
\begin{aligned}
\langle f|\mu| i\rangle= & \sum_{N_{s} \zeta} R\left(N_{t}, \zeta\right) \sum_{\mu} r_{f}^{\prime \mu \mu}(-1)^{\mu} \\
& \times\left(\begin{array}{ccc}
N_{+} & N_{i} & N_{t} \\
0 & 0 & 0
\end{array}\right)\left(\begin{array}{ccc}
N_{t} & 1 & l \\
0 & \mu & -\mu
\end{array}\right),
\end{aligned}
$$

where $N_{+}$and $N_{i}$ are the angular momenta of the ion and the resonant intermediate state (exclusive of spin), respectively. $N_{t}$ is the angular momentum transfer, $l$ is a partial wave of the photoelectron wave function, and $\mu$ is its corresponding projection on the molecular axis $(\mu=0$ and \pm 1 for the $k \sigma$ and $k \pi$ channels respectively). $R(\xi)$ and $\zeta$ represent other variables and summation indices. Details are given in Ref. 9.

The bound-free matrix element $r_{f}^{\text {l/ } \mu}$ of Eq. (4) between the intermediate state and the photoelectron continuum wave function can be written as

$$
r_{f i}^{\prime \mu \mu}=\sum_{i^{\prime}, l_{0}}\left\langle\Psi_{k l l^{\prime}}(r) Y_{l^{\prime} \lambda}\left(\hat{r}^{\prime}\right)\left|r Y_{1 \mu}\right| \Phi_{i l_{0}}(r) Y_{l_{0} 0}\left(\hat{r}^{\prime}\right)\right\rangle
$$

For a central potential $l^{\prime}=l$. However, for the noncentral potential of molecular ions, $l \neq l^{\prime}$ terms are present. These can be substantial due to the anisotropy of the molecular ion potential. This $l$ coupling in the photoelectron wave function in turn influences the ionic rotational distributions. Using the symmetry relationship $r_{f i}^{l \mu \mu}=r_{f i}^{l-\mu-\mu}$ and the properties of the 3-j symbols, ${ }^{19,20}$ it can be shown that the summation in Eq. (4) vanishes for the $\left(1+1^{\prime}\right)$ process of $\mathrm{Eq}$. (1) unless $N_{t}+N_{i}+l=$ even and $N_{t}+l=$ odd. This leads to the selection rule ${ }^{14}$

$$
N_{+}-N_{i}+l \equiv \Delta N+l=\text { odd }
$$

where $\Delta N$ is the difference between the angular momentum of the intermediate state (exclusive of spin) and the final ionic state. An atomic-like picture would predict that photoionization of the $A^{2} \Sigma^{+}(3 s \sigma)$ state should occur primarily via the $p$ wave $(3 s \rightarrow k p)$ and Eq. (6) therefore predicts a $\Delta N=$ even propensity rule.

For the electronic wave function of the intermediate $A^{2} \Sigma^{+}$state we used the improved virtual orbital approximation (IVO) ${ }^{21}$ with an extensive Gaussian basis set that ensures the correct nodal and long-range behavior of the diffuse Rydberg state. ${ }^{11}$ Single-center expansion of the $6 \sigma$ (3s $\sigma$ ) orbital of the $A^{2} \Sigma^{+}$state around the center-of-mass (c.m.) has the following partial wave composition: $94.0 \% \mathrm{~s}$, $0.2 \% p, 5.5 \% d$, and $0.1 \% f$ character. The photoelectron continuum functions were calculated using the iterative Schwinger method in the frozen-core approximation. ${ }^{22,23}$ All matrix elements are evaluated numerically for the equilibrium internuclear distance of 2.0069 a.u. of the $A^{2} \Sigma^{+}$ state. $^{24}$ The photoelectron energy in the $\left(1+1^{\prime}\right)$ REMPI experiments of Allendorf et al. ${ }^{6,7}$ is $\sim 183 \mathrm{meV}$ for the $\Delta N=0$ signal. We have previously found the bound-free matrix elements $r_{f}^{\mu \mu}$ and the ionic rotational branching ratios to be rather energy independent for REMPI via the $A^{2} \Sigma^{+}$state $^{11}$ and hence the branching ratios are all evaluated assuming a single photoelectron energy of $183 \mathrm{meV}$.

The calculated partial wave composition of the resulting photoelectron matrix elements and their relative phases are listed in Table I. These can be compared with the experimentally derived elements shown in Table IV of the preceding paper. The $p$ wave $(l=1)$ of the $k \pi$ continuum is the most dominant. The $d$ wave of the $k \sigma$ continuum is seen to be stronger than expected from the atomic picture $\left(l=l_{0}\right.$ \pm 1 ). This mixing arises from the nonisotropic nature of the molecular potential.

\section{RESULTS}

In Fig. 1 we compare the photoelectron angular distributions of Allendorf et al. ${ }^{6}$ for $\left(1+1^{\prime}\right)$ REMPI of the NO $A^{2} \Sigma^{+}$state via the rotationally clean $R_{21}$ (20.5) branch with our calculated distributions. Also shown are the angular distributions via the magic angle $\left(54.7^{\circ}\right.$ between the polarization direction of the two photons) excited $R_{21}$ (20.5) branch (see Ref. 6 for details). The intermediate state is

\begin{tabular}{|c|c|c|c|c|}
\hline \multirow[b]{2}{*}{$l$} & \multicolumn{2}{|c|}{$m=0(k \sigma$ channel $)$} & \multicolumn{2}{|c|}{$|m|=1(k \pi$ channels $)$} \\
\hline & $|r|$ & $\theta(\mathrm{rad})$ & $|r|$ & $\theta(\mathrm{rad})$ \\
\hline 0 & 0.294 & 4.88 & $\cdots$ & \\
\hline 1 & 0.519 & 2.97 & $\equiv 1.000$ & $\equiv 0.00$ \\
\hline 2 & 0.412 & 4.77 & 0.037 & 0.00 \\
\hline 3 & 0.667 & 4.78 & 0.500 & 1.61 \\
\hline 4 & 0.016 & 0.15 & 0.011 & 3.31 \\
\hline
\end{tabular}
unaligned for this excitation geometry, i.e., all $M_{J}$ levels are equally populated, and the $\beta_{4}$ coefficient of Eq. ( 3 ) is hence

TABLE I. Bound-free matrix element $\gamma_{\hat{\beta}}^{\mu \mu}$ and relative phases for a photoelectron kinetic energy of $E_{\text {kin }}=183 \mathrm{meV}$, as defined by Eq. (5). The magnitudes and phases are normalized to $l=1$ ( $p$ wave) of the $k \pi$ continuum. 


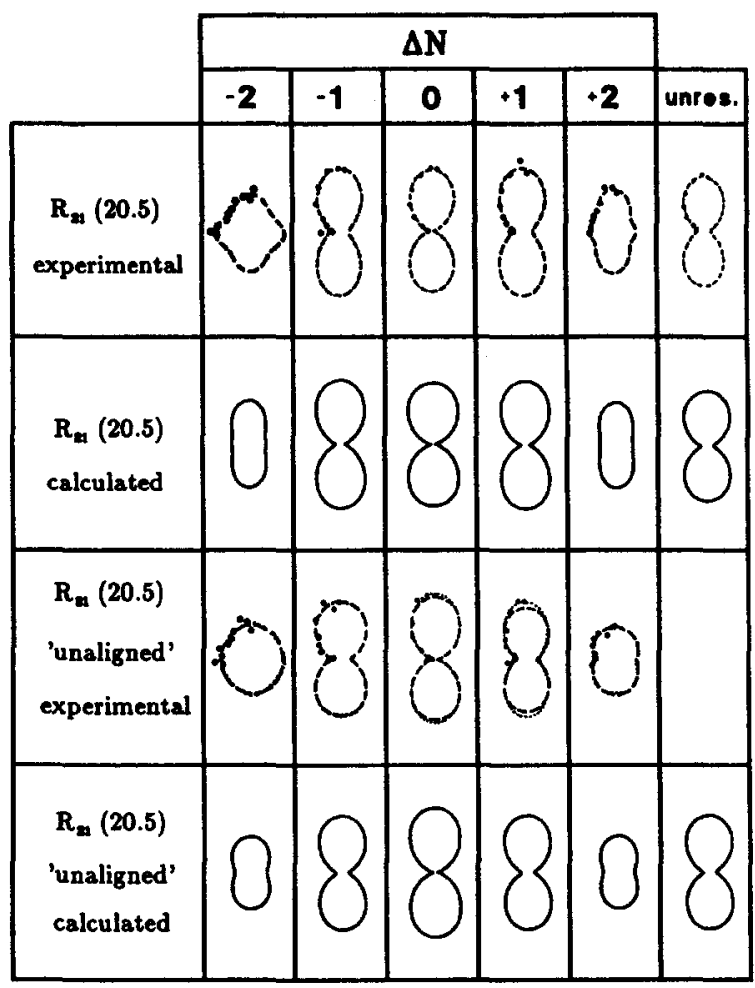

FIG. 1. Rotationally resolved $(\Delta N)$ and unresolved experimental (from Ref. 6) and calculated photoelectron angular distributions for the $R_{21}(20.5)$ and unaligned $R_{21}(20.5)$ branch, see the text for details.

zero (see Table II). The calculated and experimental photoelectron angular distributions are generally in good agreement, although there is some discrepancy for the $\Delta N=-2$ signal via the $R_{21}(20.5)$ branch for both excitation geometries. The branching ratios and angular distributions are expected to be almost symmetric around $\Delta N=0$ for these high- $J$ studies. We are not able to account for this discrepancy within the context of the present theoretical framework.

Figure 2 compares the experimental ${ }^{6}$ and calculated photoelectron angular distributions for the mixed $P_{21}+Q_{11}$ (25.5) branch. Also shown are the calculated results for the pure $P_{11}$ (22.5) branch. The agreement between the predicted and measured photoelectron angular distributions is very good for the mixed $P_{21}+Q_{11}(25.5)$ branch. Note that the angular distributions for this mixed branch

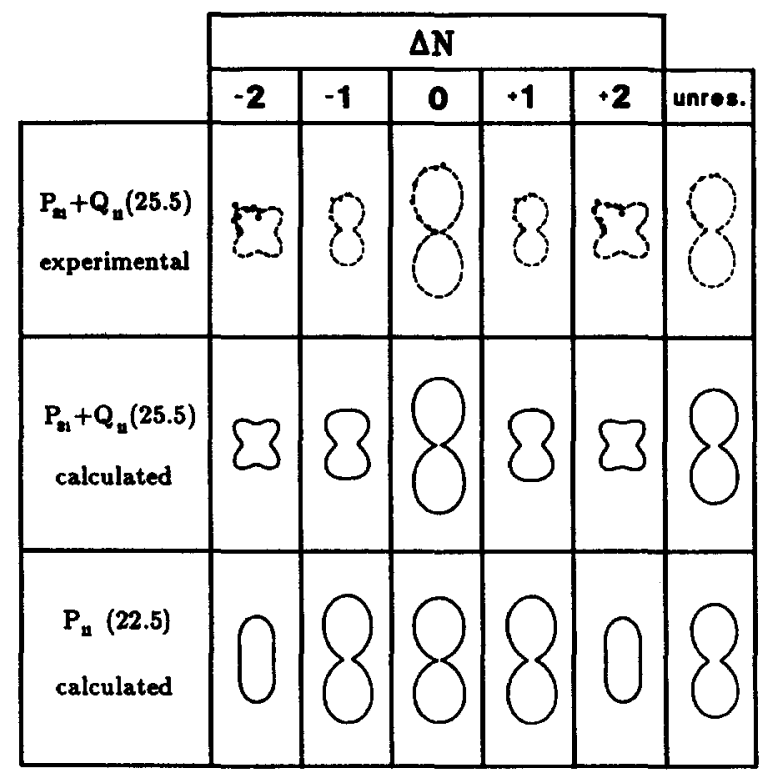

FIG. 2. Same as Fig. 1, but for the mixed $P_{21}+Q_{11}$ (25.5) branch. Also shown are the calculated photoelectron angular distributions for the pure $P_{11}$ (22.5) branch.

$\left[P_{21}+Q_{11}(25.5)\right]$ are distinctly different from those of the $R_{21}$ (20.5) branch (see Fig. 1). This reflects the importance and influence of the intermediate state's alignment on the rotationally resolved photoelectron angular distributions.

The rotationally unresolved distributions are (see Figs. 1 and 2) almost branch independent. This stresses the difficulty of probing alignment of the intermediate state with such distributions. ${ }^{25,26}$ The calculated asymmetry parameters in Table II also illustrate this since the rotationally unresolved signals are dominated by the strong $\Delta N=0$ signal. Recent rotationally unresolved $(2+1)$ REMPI experiments via the $E^{2} \Sigma^{+}$state of NO have also shown the photoelectron angular distributions to be rather insensitive to the intermediate state's alignment, ${ }^{25}$ in agreement with the present case. Theoretical calculations confirm that the rotationally unresolved angular distributions via the $E$ state are alignment independent and that the rotationally resolved distributions are very branch dependent. ${ }^{26}$

The model employed by Allendorf $e t a l .^{6}$ to fit the experimental angular distributions as in Figs. 9-12 of Ref. 6, is

TABLE II. Calculated asymmetry parameters $\left(\beta_{2 L}\right)$ for the $R_{21}(20.5), R_{21}(20.5)$ (unaligned),

$P_{21}+Q_{11}$ (25.5), and the $P_{11}(22.5)$ branches, as defined by Eq. (3), with $\beta_{0} \equiv 1.000$.

\begin{tabular}{|c|c|c|c|c|c|c|c|c|}
\hline \multirow[b]{2}{*}{$\Delta N$} & \multicolumn{2}{|c|}{$R_{21}(20.5)$} & \multicolumn{2}{|c|}{$R_{21}(20.5)^{a}$} & \multicolumn{2}{|c|}{$P_{21}+Q_{11}(25.5)$} & \multicolumn{2}{|c|}{$P_{11}(22.5)$} \\
\hline & $\beta_{2}$ & $\beta_{4}$ & $\beta_{2}$ & $\beta_{4}$ & $\beta_{2}$ & $\beta_{4}$ & $\beta_{2}$ & $\beta_{4}$ \\
\hline-2 & 0.676 & 0.251 & 0.580 & 0.000 & 0.387 & -0.507 & 0.665 & 0.222 \\
\hline-1 & 1.724 & 0.111 & 1.492 & 0.000 & 0.824 & -0.318 & 1.699 & 0.100 \\
\hline 0 & 1.794 & -0.068 & 1.835 & 0.000 & 1.886 & 0.087 & 1.800 & -0.059 \\
\hline+1 & 1.730 & 0.099 & 1.492 & 0.000 & 0.829 & -0.280 & 1.704 & 0.088 \\
\hline+2 & 0.666 & 0.222 & 0.580 & 0.000 & 0.409 & -0.445 & 0.655 & 0.195 \\
\hline $\begin{array}{l}\text { Rot. } \\
\text { unres. }\end{array}$ & 1.458 & 0.027 & 1.486 & 0.000 & 1.548 & -0.040 & 1.482 & 0.024 \\
\hline
\end{tabular}

" "Unaligned" (54.7\%), see the text for details. 
TABLE III. Calculated angle-integrated rotational branching ratios normalized to the $\Delta N=0$ signal.

\begin{tabular}{rcccc}
\hline \hline$\Delta N$ & $R_{21}(20.5)$ & $R_{21}(20.5)^{\mathrm{a}}$ & $P_{21}+Q_{11}(25.5)$ & $P_{11}(22.5)$ \\
\hline-2 & 0.199 & 0.167 & 0.126 & 0.194 \\
-1 & 0.053 & 0.040 & 0.024 & 0.051 \\
0 & $\equiv 1.00$ & $\equiv 1.00$ & $\equiv 1.00$ & $\equiv 1.00$ \\
+1 & 0.055 & 0.042 & 0.025 & 0.053 \\
+2 & 0.216 & 0.182 & 0.138 & 0.211 \\
\hline \hline
\end{tabular}

a "Unaligned" $\left(54.7^{\circ}\right)$, see the text for details.

based on the same theoretical framework used in our analysis. The photoelectron matrix elements are treated there as adjustable fitting parameters. ${ }^{6}$ Within this model these branching ratios and angular distributions should be almost symmetric around $\Delta N=0$. The asymmetry of the $\Delta N=\mp 2$ angular distributions for the $R_{21}$ (20.5) branch in Fig. 5 of Ref. 6 can therefore not be accounted for on the basis of the present model or fits based on it. The asymmetry between the measured and the "model" fits must therefore be due to perturbations not included in the model or to experimental uncertainties.

Table III lists the calculated angle-integrated ionic rotational branching ratios. The $\Delta N=0$ signal is dominant for all branches and is more than five times stronger than the $\Delta N= \pm 2$ signals and $\sim 20$ times stronger than the $\Delta N= \pm 1$ signals. These $\Delta N= \pm 1$ signals had not been resolved in earlier experiments, ${ }^{4,5}$ but were predicted theoretically ${ }^{9,11}$ to be $\sim 5 \%$ of the $\Delta N=0$ signal. This agrees well with the experimental results.

\section{CONCLUSION}

We have reported the ionic rotational branching ratios and photoelectron angular distributions for low photoelectron energies resulting from $\left(1+1^{\prime}\right)$ REMPI via the $R_{21}$ (20.5), $P_{11}$ (22.5), and $P_{21}+Q_{11}$ (25.5) branches of the $A^{2} \Sigma^{+}(3 s \sigma)$ state of NO. Comparison with the experimental high resolution experimental results of Allendorf et al.$^{6,7}$ shows quantitative agreement between the calculated and measured values. The ionic rotational branching ratios are seen to be weakly branch dependent. However, the rotationally resolved photoelectron angular distributions are very branch (alignment) dependent, whereas the rotationally unresolved ones are less so. This makes it difficult to probe the alignment of intermediate states via rotationally unresolved measurements. The rotationally resolved photoelectron angular distributions are clearly important for elucidating the dynamical details of the REMPI process.

\section{ACKNOWLEDGMENTS}

This material is based on research supported by the National Science Foundation under Grant No. CHE-8521391, by AFOSR under Grant. No. 87-0039 and by the Office of Health and Environmental Research of DOE (DE-FG0387ER60513). The authors also acknowledge use of the resources of the San Diego Supercomputer which is supported by the National Science Foundation. H.R. gratefully acknowledges support from the NATO Science Fellowship Programme.

'S. T. Pratt, P. M. Dehmer, and J. L Dehmer, Chem. Phys. Lett. 105, 28 (1984).

${ }^{2}$ K. Kimura, in Photodissociation and Photoionization (Wiley, New York, 1985), p. 161 and references therein

${ }^{3}$ S. T. Pratt, P. M. Dehmer, and J. L. Dehmer, J. Chem. Phys. 78, 4315 (1983).

${ }^{4}$ W. G. Wilson, K. S. Viswanathan, E. Sekreta, and J. P. Reilly, J. Phys. Chem. 88, 672 (1984)

${ }^{5}$ K. S. Viswanathan, E. Sekreta, E. R. Davidson, and J. P. Reilly, J. Phys. Chem. 90, 5078 (1986).

${ }^{6} \mathrm{~S}$. W. Allendorf, D. J. Leahy, D. C. Jacobs, and R. N. Zare, J. Chem. Phys. 91, 2216 (1989).

${ }^{7}$ S. W. Allendorf, Bull. Am. Phys. Soc. 33, 920 (1988).

${ }^{8}$ V. McKoy, M. Braunstein, S. N. Dixit, D. L. Lynch, H. Rudolph, and J. Stephens, Bull. Am. Soc. Phys. 33, 919 (1988).

${ }^{9}$ S. N. Dixit, D. L. Lynch, V. McKoy, and W. M. Huo, Phys. Rev. A 32, 1267 (1985)

${ }^{10}$ H. Rudolph, S. N. Dixit, V. McKoy, and W. M. Huo, Chem. Phys. Lett. 137, 521 (1987)

'H. Rudolph, S. N. Dixit, V. McKoy and W. M. Huo, J. Chem. Phys. 88, 637 (1988).

${ }^{12}$ H. Rudolph, S. N. Dixit, V. McKoy, and W. M. Huo, J. Chem. Phys. 88, 1516 (1988).

${ }^{13}$ H. Rudolph, S. N. Dixit, and V. McKoy, J. Chem. Phys. 90, 2570 (1989).

${ }^{14}$ S. N. Dixit and V. McKoy, Chem. Phys. Lett. 128, 49 (1986).

${ }^{15}$ X. Song, E. Sekreta, J. P. Reilly, H. Rudolph, and V. McKoy (to be published).

${ }^{16}$ M. Sander, L. A. Chewter, K. Müller-Dethlefs, and E. W. Schlag, Phys. Rev. A 36, 4543 (1987).

${ }^{12}$ S. N. Dixit and V. McKoy, J. Chem. Phys. 82, 3546 (1985).

${ }^{18}$ L. T. Earls, Phys. Rev. 48, 423 (1935).

${ }^{19}$ A. R. Edwards, Angular Momentum in Quantum Mechanics, 2 nd ed. (Princeton University, Princeton, N.J., 1974).

${ }^{20}$ R. N. Zare, Angular Momentum, Understanding Spatial Aspects in Chemistry and Physics (Wiley, New York, 1988).

${ }^{21}$ W. J. Hunt and W. A. Goddard III, Chem. Phys. Lett. 24, 464 (1974).

${ }^{22}$ R. R. Lucchese and V. McKoy, Phys. Rev. A 21, 112 (1980).

${ }^{23}$ R. R. Lucchese, G. Raseev, and V. McKoy, Phys. Rev. A 25, 2572 (1982).

${ }^{24} \mathrm{~K}$. P. Huber and G. Herzberg, Constants of Diatomic Molecules (Van Nostrand Reinhold, New York, 1979).

${ }^{25}$ J. R. Appling, M. G. White, W. J. Kessler, R. Fernandez, and E. D. Poliakoff, J. Chem. Phys. 88, 2300 (1988).

${ }^{26} \mathrm{H}$. Rudolph and V. McKoy, J. Chem. Phys. (to be published). 